\title{
Brainstem disconnection
}

\author{
Curtis Duffield • Jennifer Jocson • \\ Sandra L. Wootton-Gorges
}

Received: 2 June 2009 /Revised: 9 July 2009 / Accepted: 28 July 2009 / Published online: 11 August 2009

(C) The Author(s) 2009. This article is published with open access at Springerlink.com

\begin{abstract}
Brainstem disconnection is a very rare neonatal abnormality, with only seven cases reported. We report a unique case of a neonate who presented at delivery with hypertonia, dysmorphic facial features, and respiratory distress, as well as numerous musculoskeletal and genitourinary abnormalities. MRI of the brain showed disconnection between the pons and medulla with cerebellar hypoplasia and absent cerebellar peduncles. It aided in the description of the neurological and vascular anomalies associated with this diagnosis.
\end{abstract}

Keywords Brainstem · Congenital malformation · MRI · Neonate

\section{Introduction}

Brainstem disconnection is a rare entity described only in the neonate. Seven prior neonates have been reported in the English literature, with all dying by 7 weeks of age [1]. The etiology of brainstem disconnection remains uncertain. Proposed theories include variants of pontocerebellar hypoplasia or other mid- and hindbrain malformations (such as Joubert syndrome, Mobius syndrome, etc.) as well as vascular insult with brainstem necrosis [1-3].

C. Duffield · S. L. Wootton-Gorges $(\bowtie)$

Department of Radiology, University of California Davis, Medical Center and UC Davis Children's Hospital,

4860 Y St., Suite 3100 ,

Sacramento, CA 95817, USA

e-mail: sandra.gorges@ucdmc.ucdavis.edu

\section{J. Jocson}

Department of Pediatrics, University of California Davis, Medical Center and UC Davis Children's Hospital,

Sacramento, CA, USA
Brainstem disconnection is generally unrecognized prenatally, although cerebellar hypoplasia can be seen on prenatal US $[1,2]$. Neonatal clinical findings vary but generally involve dense neurologic dysfunction. Advances in imaging, especially MRI, have allowed more detailed evaluation of the anatomic anomalies that make up this disorder.

We present MRI and MRA imaging of brainstem disconnection with neurologic and vascular anomalies, as well as extracerebral malformations. A discussion of brainstem disconnection, its proposed etiologies, and its differential diagnoses are given.

\section{Case report}

A girl was born at term to a 23-year-old woman. A secondtrimester US had shown bilateral ventriculomegaly, left hydronephrosis, a 2-vessel cord, vertebral segmentation anomalies, and polyhydramnios. At delivery, the infant was dysmorphic and had a poor cry and increased tone with jitteriness. The infant weighed 3,125 g (35th percentile), and had an occipital-frontal circumference of $35.5 \mathrm{~cm}$ (65th percentile). Physical examination revealed right microophthalmia, hypertelorism, an asymmetrically smaller right face with elevated nasal bridge, and a thin and downturned upper lip. A lower lumbar deformity was noted as well as a heart murmur. The neurologic exam found hypertonia, cortical thumb posturing, poor suck, exaggerated reflexes and seizure-like activity similar to myoclonic jerks. EEG showed abnormal baseline activity but did not specifically correlate with the myoclonic jerks. Echocardiogram showed only a small muscular ventricular septal defect. High-resolution chromosome studies were normal. 

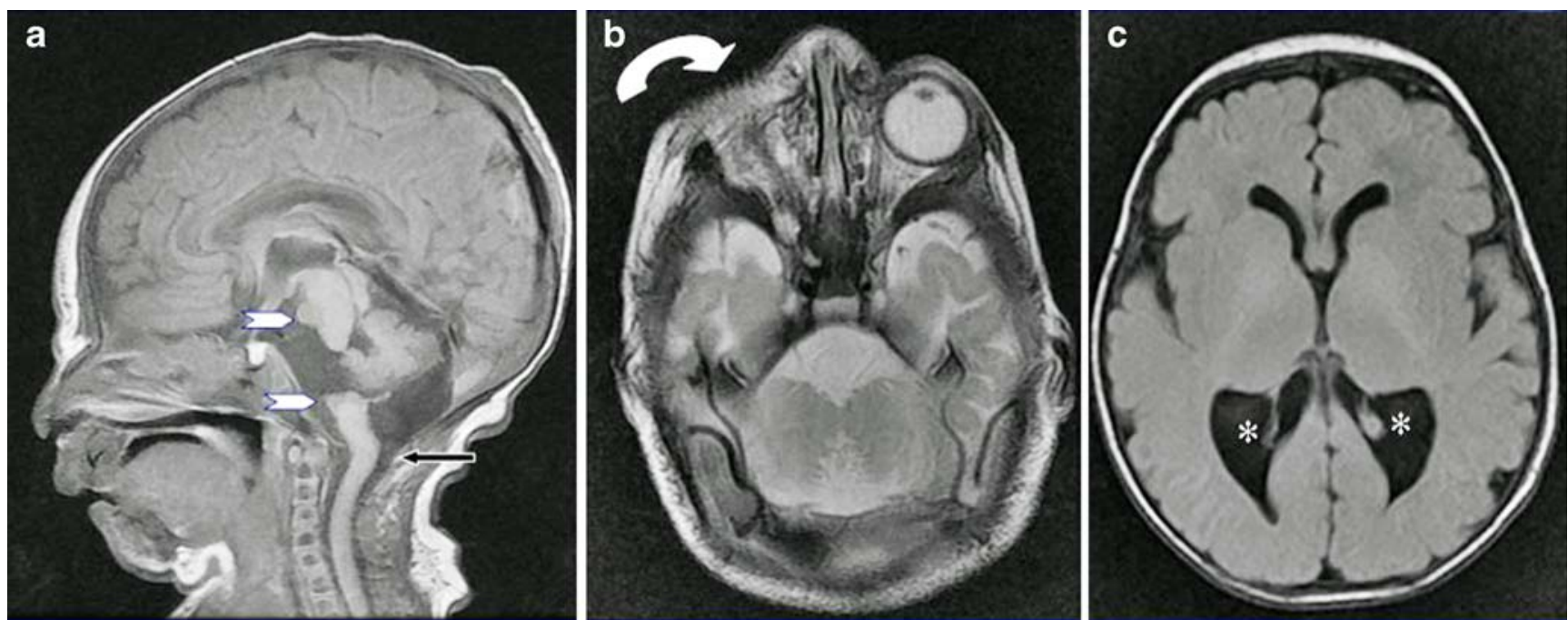

Fig. 1 Brain MRI at 1 day old. Sagittal T1-W (a) and axial T2-W (b) images show disconnection between the midbrain and medulla with absence of the pons (between white arrowheads). Plate-like ectopic neuronal tissue/white matter is seen at the ventral aspect of the midbrain and medulla (arrowheads). There is absence of the vermis, cerebellar hemisphere symmetrical hypoplasia and absence of the cerebellar peduncles, and a prominent cisterna magna. There is kinking of the brainstem (black arrow). Colpocephaly (*) is noted on axial T2-inversion-recovery images (c). Additional findings include right anophthalmia with an absent right optic nerve (curved arrow)
MRI of the brain on day 1 demonstrated brainstem disconnection between the midbrain and medulla with absence of the pons (Fig. 1). No tissue cord was seen connecting the midbrain and medulla. Plate-like ectopic neuronal tissue/white matter, perhaps representing pontine remnants, was seen at the ventral aspect of the pontomidbrain junction and at the superior ventral medulla. There was absence of the vermis and symmetrical moderate cerebellar hemisphere hypoplasia, absence of the inferior and middle cerebellar peduncles, colpocephaly, a distorted 4th ventricle and a prominent mega cisterna magna. Additional findings included a hypoplastic corpus cal- losum, a small hypothalamus, a distorted tectum, and right anophthalmia with an absent right optic nerve.

MR angiography showed absence of the majority of the basilar artery (Fig. 2). Small vertebral arteries were seen to the foramen magnum and joined to form a tiny proximal basilar artery, from which a tiny right posterior inferior cerebellar artery was seen. Further definition of the posterior fossa vascular anatomy was technically limited because of the small patient size. The carotid arteries and circle of Willis were intact with patent posterior communicating arteries and P1 segments of the posterior cerebral arteries. MR venography was normal.
Fig. 2 Angiography. Sagittal (a) and axial (b) MIP 3-D timeof-flight MRA show absence of the basilar artery (white arrow). The carotid arteries and circle of Willis are intact with patent posterior communicating arteries and P1 segments of the posterior cerebral arteries (arrowheads). A tiny right PICA is seen. MR venography was normal
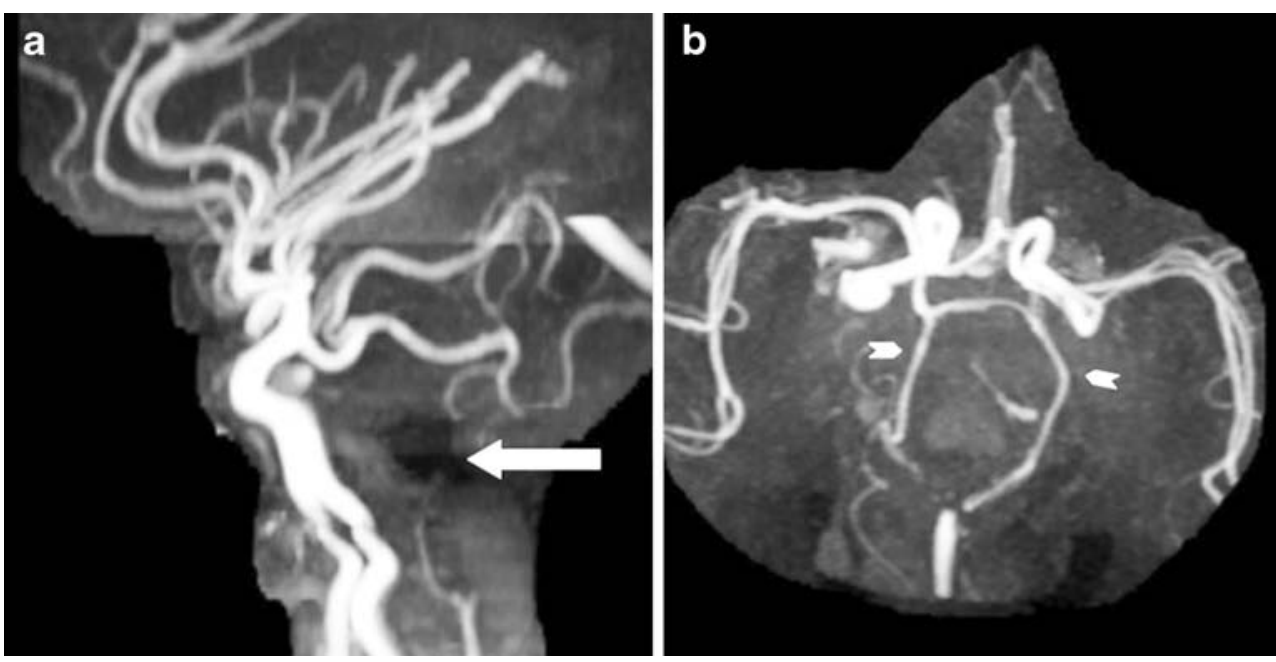
Plain radiography demonstrated multiple thoracic spine segmentation anomalies and hypoplasia of the distal sacrum and coccyx. MR of the spine demonstrated kinking of the brainstem but otherwise normal cord contour and signal. The conus was at the L2-3 level.

Neonatal abdominal US demonstrated grade 3 left hydronephrosis and hydroureter. A voiding cystourethrogram showed grade 4-5 left vesicoureteral reflux. A bicornuate uterus was also noted at MR.

Because of the poor prognosis she was referred to hospice and discharged at 35 days old. She died at 8 weeks of age. No autopsy was performed.

\section{Discussion}

Brainstem disconnection is a rare neonatal congenital abnormality. Only seven cases have been described in the English literature. All were fatal by 7 weeks of age [1-5]. The disconnection can be between the midbrain and pons (pontomesencephalic) or between the pons and medulla (pontomedullary) [1]. Associated neurological anomalies include vermian and cerebellar hypoplasia [1], microcephaly, and absence of the basilar artery [3]. One case also described associated optic nerve hypoplasia [2]. Extracerebral anomalies are less common. Two cases of cardiac defects and one case of ectopic thyroid and parathyroid tissue associated with a small thymus have been reported [1].

Pregnancy has been full-term in all cases, sometimes complicated by polyhydramnios [1]. Antenatal imaging, including sonography and fetal MRI, has occasionally described cerebellar hypoplasia and pontine hypoplasia [2] but has failed to detect the brainstem disconnection. Presenting clinical findings at birth might include hypotonia or hypertonia, absent or weak suck reflex, seizures, and respiratory difficulties.

The etiology of brainstem disconnection is uncertain. It is also unclear whether the two disconnection locations result from different pathologic or genetic etiologies. In a murine model, pontomesencephalic disconnection was related to mutation in the EN2 gene [4]. However, no genetic mutations have been detected in humans.

The topic of brainstem malformations is complex. Barkovich, Millen and Dobyns [6] have proposed a classification of these malformations based upon their embryological mechanism of development while Parisi and Dobyns [7] proposed a classification based upon their anatomic derivation. The development of the pons is intricately woven to the medulla and cerebellum. Arising from the rhombencephalon, its differentiation takes place between 34 and 42 days of gestation and is susceptible to numerous influences that can cause structural defects [2]. Abnormal induction, cell specification, proliferation or apoptosis can lead to hypoplasia of the pons [6]. Neuronal path-finding abnormalities can result in associated pontine clefts. Cerebellar hypoplasia is commonly associated with pontine hypoplasia [6]. Additionally, it has been proposed that insults in the 2nd and 3rd trimesters can also result in brainstem/pontocerebellar hypoplasia and can be associated with neuronal loss and gliosis [8].

Others have suggested that brainstem disconnection is at one end of the spectrum of pontoneocerebellar hypoplasia. In this abnormality there is severe reduction in the number of neurons in the pontine nuclei, in transverse pontine fibers, in the middle cerebellar peduncles and lateral cerebellar lobes, and in inferior olivary, arcuate and dentate nuclei [8]. Progressive atrophy occurs over time [7].

A vascular insult to the basilar artery has been proposed as an etiological mechanism for pontomedullary disconnection [3]. Supporting this, several cases have documented absence of basilar artery flow. In addition, focal brainstem necrosis has been described following severe hypotension and cardiac arrest. However, complete absence of the brainstem has not been described in such cases. Further, no histological evidence of previous brainstem infarction has been found in cases of brainstem disconnection [4].

Brainstem disconnection differs from other more familiar brainstem malformations. Patients with Joubert syndrome have a normal pons, a deep midbrain interpeduncular fossa, horizontal superior cerebellar peduncles, and cerebellar vermian hypoplasia/aplasia resulting in the "molar tooth" sign $[6,7]$. Patients with Möbius syndrome have 6th and 7th cranial nuclear aplasia resulting in a small dorsal pons. These patients might have associated limb, facial and chest wall abnormalities. Patients with congenital muscular dystrophy with O-glycosylation defects (Walker-Warburg syndrome, muscle-eye-brain disease, Fukuyama congenital muscular dystrophy, etc.) present in infancy with failure to thrive, hypotonia and ataxia. They have dysmorphic facies, lipodystrophy, and inverted nipples [7]. The entire brainstem is thin, with a midbrain-pontine junction kink and a small ventral pontine cleft. The cerebellum is small and dysmorphic [6].

Our case differs from previous cases because of the presence of multiple extracerebral manifestations, including spinal segmentation and rib anomalies, genitourinary anomalies (hydronephrosis/hydroureter and bicornuate uterus), ventricular septal defect, and ocular malformation (right anophthalmia and optic nerve aplasia). This constellation of findings does not fit into any currently described syndromes of which we are aware.

In summary, we offer imaging and discussion of a rare case of brainstem disconnection. The etiology of this malformation is unclear, but the leading theories have been discussed. Our case is unique in that this baby girl also presented with multiple extracerebral associated malformations. 
Open Access This article is distributed under the terms of the Creative Commons Attribution Noncommercial License, which permits any noncommercial use, distribution, and reproduction in any medium, provided the original author(s) and source are credited.

\section{References}

1. Poretti A, Boltshauser E, Plecko B (2007) Brainstem disconnection: case report and review of the literature. Neuropediatrics 38:210-212

2. McCann E, Pilling D, Hesseling M et al (2005) Pontomedullary disconnection: fetal and neonatal considerations. Pediatr Radiol 35:812-814

3. Mamourian A, Miller G (1994) Neonatal pontomedullary disconnection with aplasia or destruction of the lower brain stem: a case of pontoneocerebellar hypoplasia? AJNR 15:14831485

4. Sarnat HB, Benjamin DR, Siebert JR et al (2002) Agenesis of the mesencephalon and metencephalon with cerebellar hypoplasia: putative mutation in the EN2 gene-report of 2 cases in early infancy. Pediatr Dev Pathol 5:54-68

5. Bednarek N, Scavarda D, Mesmin F et al (2005) Midbrain disconnection: an aetiology of severe central neonatal hypotonia. Eur J Paediatr Neurol 9:419-422

6. Barkovich AJ, Millen KJ, Dobyns WB (2007) A developmental classification of malformation of the brainstem. Ann Neurol 62:625-639

7. Parisi MA, Dobyns WB (2003) Human malformations of the midbrain and hindbrain: review and proposed classification scheme. Mol Genet Metab 80:36-53

8. Pittella JE, Nogueira AM (1990) Pontoneocerebellar hypoplasia: report of a case in a newborn and review of the literature. Clin Neuropathol 9:33-38 